\title{
Mathematical Models of Dynamic Behavior of Individual Neural Networks of Central Nervous System
}

\author{
Dimitra-Despoina Pagania ${ }^{1, *}$, Adam Adamopoulos ${ }^{1,2}$, \\ and Spiridon D. Likothanassis ${ }^{1}$ \\ ${ }^{1}$ Pattern Recognition Laboratory, Dept. of Computer Engineering \& Informatics, \\ University of Patras, 26500, Patras, Greece \\ ${ }^{2}$ Dept. of Medicine, Democritus University of Thrace, Alexandroupolis, Greece \\ \{pagania, likothan\} @ceid.upatras.gr, adam@med.duth.gr
}

\begin{abstract}
We present mathematical models that describe individual neural networks of the Central Nervous System. Three cases are examined, varying in each case the values of the refractory period and the synaptic delay of a neuron. In the case where both the refractory period and the synaptic delay are bigger than one, we split the population of neurons into sub-groups with their own distinct synaptic delay. It is shown that the proposed approach describes the neural activity of the network efficiently, especially in the case mentioned above. Various examples with different network parameters are presented to investigate the network's behavior.
\end{abstract}

Keywords: refractory period, synaptic delay, neural activity, mathematical models, asynchronous synapses.

\section{Introduction}

In this paper we describe and analyze mathematical models that are used for the statistical behavior of individual -without external links- neural networks of the Central Nervous System. If a neuron is activated at some point then a certain moment is necessary to pass for the neuron to be able to spike again. We consider this moment as the refractory period (r). Furthermore, we consider the moment that mediates between the spike of a neuron until the appearance of postsynaptic potential (PSP) [5] at the postsynaptic neuron being the synaptic delay $(\tau)$ [1], [2], [3]. Initially, we investigate the case where the refractory period is set to $1(\mathrm{r}=1)$ and the synaptic delay is set to 1 too $(\tau=1)$. The second under investigation case deals with individual neural networks with excitatory synapses where the refractory period is bigger than $1(r>1)$ while the synaptic delay is set to $1(\tau=1)$ like in the first case. The third and last case studies asynchronous individual neural networks with excitatory synapses where both the refractory period and the synaptic delay are bigger than $1(r>1, \tau>1)$ [4]. Despite the fact that the last years have seen a dramatic increase of computer simulation models that were developed to investigate the issue of structure and function of the human

\footnotetext{
* Corresponding author.
} 
brain [6], [7] a few have appeared concerning neural network models with asynchronous neuron. By this term we mean the investigation of the dynamical behavior of neural network models consisting of neurons that are able to fire and cease at different time instants and not at the same time, as it is the case in neural networks consisting of synchronous neurons.

\section{Individual Neural Networks with Excitatory Synapses}

We study an individual neural network consisted of $N$ neurons [4]. Each neuron has $\mu$ synapses, triggering threshold $\theta$ and refractory period $\mathrm{r}$. Each synapse has synaptic weight w. All N neurons product Post Synaptic Potential (PSP) in case they are excited after $\tau$ time steps. We consider, for the moment being, that $r=1$ and $\tau=1$ committing that we will investigate the more general cases of $r \geq 1$ and $\tau \geq 1$ later on. If at moment $n$ the percentage of active neurons of the network is $\alpha_{n}$ the question is to find the corresponding percentage of active neurons at the moment $n+1$, meaning to find the $\alpha_{n+1}$ versus $\alpha_{n}$. If $\alpha_{n}$ is the percentage of active neurons at moment $n$, then the number of active neurons at that moment is $N \times \alpha_{n}$. Thus, at the moment $\mathrm{n}+1, L=N \times$ $\alpha_{n} \times \mu$ PSPs should be produced. We have to calculate the probability for a neuron to receive a total PSP that is either greater or equal to triggering threshold $\theta$ so that it is able to spike. This $\mathrm{p}_{\mathrm{k}}$ probability is calculated by the binomial distribution

$$
p_{k}=\left(\begin{array}{l}
L \\
k
\end{array}\right)\left(\frac{1}{N}\right)^{k}\left(1-\frac{1}{N}\right)^{L-k} .
$$

where L are the total PSPs produced by the active neurons and $\mathrm{k}$ are the ones that the neuron will receive. The neuron will be excited if $k \times w \geq \theta$ or $k \geq \theta / w$. If the synaptic weight $\mathrm{w}$ is set to 1 , the last treaty is transformed to $k \geq \theta$. Therefore, the probability for a neuron to receive a total PSP either greater or equal to triggering threshold $\theta$ equals to the sum of $\mathrm{p}_{\mathrm{k}}$ probabilities for $k \geq \theta$. Thus,

$$
P\left(a_{n}, \theta\right)=\sum_{k=\theta}^{L} p_{k} .
$$

We must now take into consideration the refractory period $r$ of the neurons. At the moment $\mathrm{n}$, the number of active neurons is $N \times \alpha_{n}$. If $\mathrm{r}=1$ then at the next time step, at the moment $\mathrm{n}+1$, the number of neurons that are able to spike is

$$
N-N \times \alpha_{\mathrm{n}}=N \times\left(1-\alpha_{\mathrm{n}}\right) .
$$

and the percentage of these neurons is

$$
\alpha_{n+1}=\left(1-\alpha_{n}\right) \times P\left(\alpha_{n}, \theta\right) \quad .
$$

dividing by the number of neurons $\mathrm{N}$ the equation (3). Based on the above, we investigate the behavior of the neural network varying the following parameters: 1 . Number of neurons N, 2. Number of synapses of each neuron $\mu$, 3. Triggering threshold of each neuron $\theta$, 4. Synaptic weight w. Specifically, we calculate the 
percentage of neurons that will be activated at the moment $n+1, \alpha_{n+1}$, and the percentage of neurons that are active at the moment $n, \alpha_{n}$. Also, in each case we calculate the steady states and with every change of the parameters. By steady states we mean the case where $\alpha_{n}=\alpha_{n+1}$.

\section{Changes at the Network's Behavior}

\subsection{Varying the Number of Neurons $\mathbf{N}$}

We maintain the following values for $\mu=70, \theta=15$, w $=0.8$. In table 1 the steady states are presented.

Table 1. Steady states for number of neurons N

\begin{tabular}{llr}
\hline Number of Neurons N & \multicolumn{2}{l}{ Steady States } \\
\hline 10 & 0.2000 & 0.4995 \\
200 & 0.1700 & 0.5000 \\
5000 & 0.1670 & 0.5000 \\
10.000 & 0.2378 & 0.4997 \\
$10^{11}$ & 0.1668 & 0.5000 \\
\hline
\end{tabular}

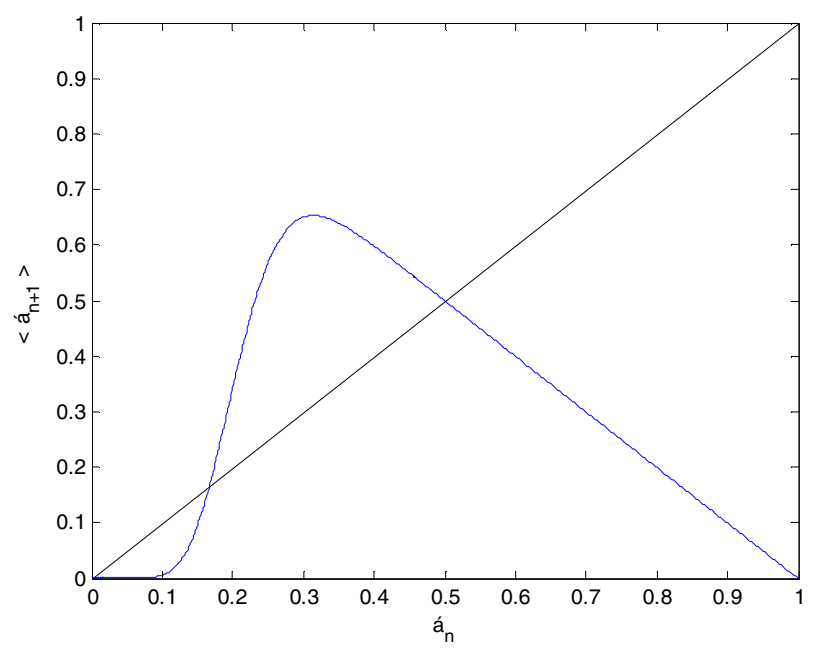

Fig. 1. We choose the number of neurons $N$ to be 10000 . While $\alpha_{n}$ increases, $\alpha_{n+1}$ increases too, until it reaches a percentage of the order of 0.7 where it decreases. We mention that for arbitrarily big number of neurons we choose to investigate, the behavior is still the same.

\subsection{Varying the Number of Synapses $\mu$ per Neuron}

We maintain the following values for $\mathrm{N}=10.000, \theta=15, \mathrm{w}=0.8$. In table 2 the steady states are presented. 
Table 2. Steady states for number of synapses $\mu$

\begin{tabular}{lll}
\hline Number of Synapses $\mu$ Per Neuron & \multicolumn{2}{l}{ Steady States } \\
\hline 15 & {[]} & \\
70 & 0.2376 & 0.4997 \\
100 & 0.1493 & 0.5000 \\
500 & 0.0227 & 0.5000 \\
1000 & 0.0105 & 0.5000 \\
7000 & 0.0009 & 0.5000 \\
\hline
\end{tabular}

It is obvious that there are no steady states when the number of synapses per neuron is small. In this case, the neurons that could be activated at the moment $n+1$ do not activate at all -only a small percentage- and only when the percentage of neurons activated at the moment $n$ is relatively big. That means that the number of synapses per neuron affects the $\alpha_{n+1}$ (the $\alpha_{n}$ too). As $\mu$ is increased the steady states correspond to small percentages of neurons that are activated at the moment $n$. That means that when each neuron is concluded with many neurons or when it has many synapses with each neuron of the network, not a big number of neurons are necessary to be activated at the moment $\mathrm{n}$ so that the neurons at the next time step, $\mathrm{n}+1$, are activated.

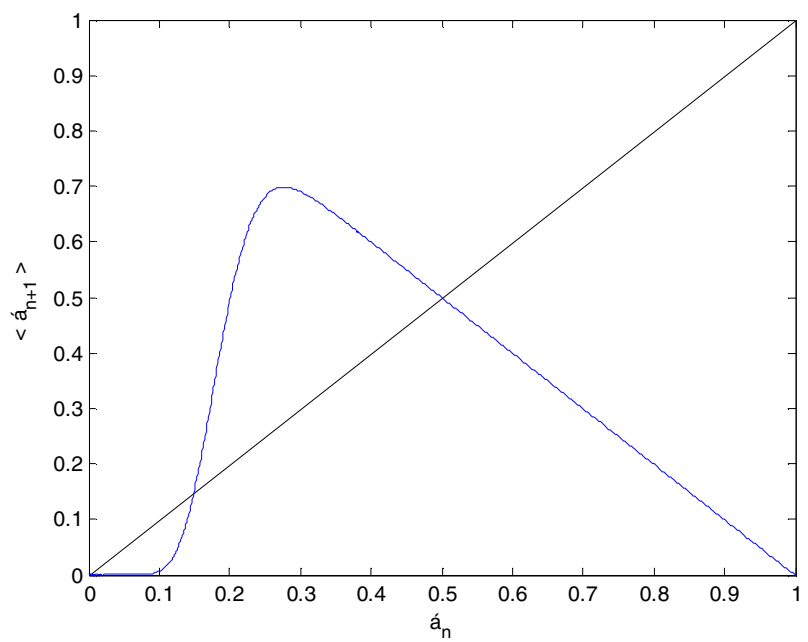

Fig. 2. When the percentage of neurons activated at the moment $n$ overcome the 0.1 value, the percentage of neurons activated at the moment $n+1$ increases rapidly until it reaches a maximum limit $(=\sim 0.71)$ after which it decreases.

For a big number of synapses per neuron the behavior of the network is radically different than the case where e.g. $\mu=15$. The steady states are two and the first one as already mentioned- corresponds to the activation of a small percentage of neurons. 


\subsection{Varying the Triggering Threshold of Neurons $\theta$}

We maintain the following values for $\mathrm{N}=10.000, \mu=70, \mathrm{w}=0.8$. In table 3 the steady states are presented.

Table 3. Steady states for triggering threshold of neurons

\begin{tabular}{lll}
\hline Triggering Threshold of Neurons $\theta$ & \multicolumn{3}{l}{ Steady States } \\
\hline 5 & 0.0468 & 0.5000 \\
9 & 0.1186 & 0.5000 \\
15 & 0.2376 & 0.4997 \\
30 & {[]} & \\
70 & {[]} & \\
\hline
\end{tabular}

As the triggering threshold of neurons is increased we reach to a point where there are no steady states since the mathematical treaty $\mathrm{k} \geq \theta / \mathrm{w}$ is no longer valid. The smaller the threshold $\theta$, the faster the $\alpha_{n+1}$ is increased versus $\alpha_{n}$ and steady states exist for a rather small percentage of activated neurons.

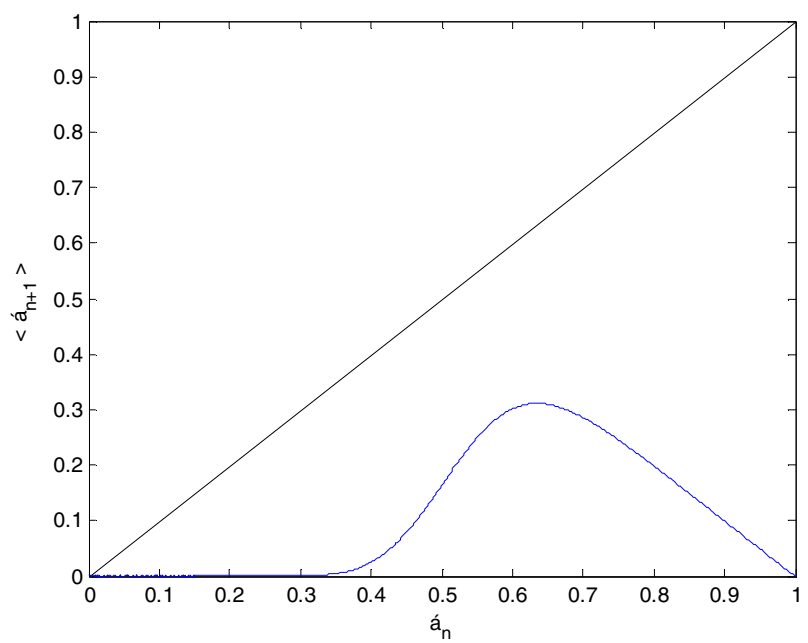

Fig. 3. For $\theta=30 . \alpha_{n+1}$ is set to 0 for a percentage of neurons equal to or bigger than 0.33 . That means that neurons are not activated at all since $\mathrm{k} \geq \theta / \mathrm{w}$ is no longer valid and $\theta$ is relatively big.

\subsection{Varying the Synaptic Weight w}

We maintain the following values for $\mathrm{N}=10.000, \mu=70, \theta=15$. In table 4 the steady states are presented. 
Table 4. Steady states for synaptic weight w

\begin{tabular}{llll}
\hline Synaptic weight & $\mathrm{w}$ & \multicolumn{2}{l}{ Steady States } \\
\hline 0.1 & & {[]} & \\
0.6 & 0.3668 & 0.4892 \\
1.5 & 0.0883 & 0.5000 \\
\hline
\end{tabular}

Again, since $\mathrm{k} \geq \theta / \mathrm{w}$ and $\theta$ is stable, as synaptic weight $\mathrm{w}$ is increased, the percentage of activated neurons at the moment $n+1$ is increased too. Contrary, for a small $w$, e.g. $\mathrm{w}=0.1, \alpha_{\mathrm{n}+1}$ is set to 0 no matter how many neurons are activated at the moment n. Of course, in this case there are no steady states for the network.

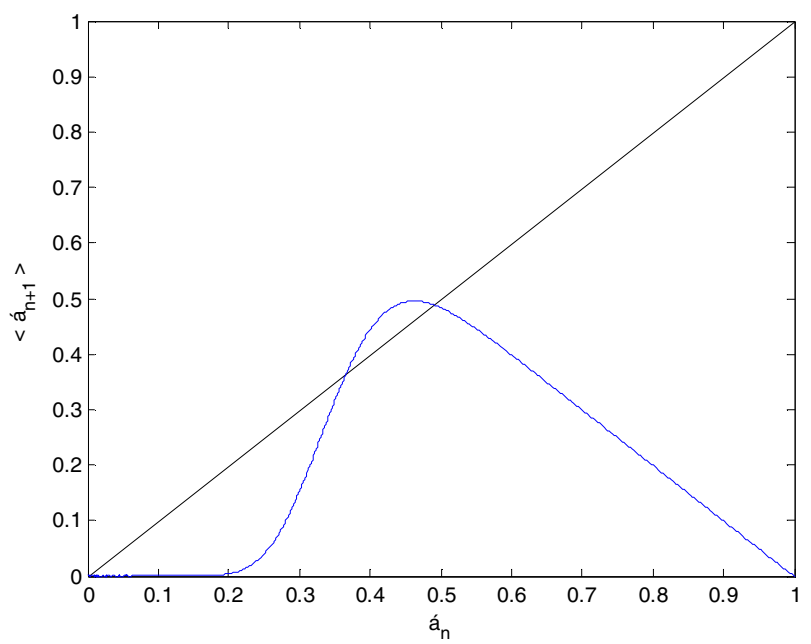

Fig. 4. For $w=0.6$. By setting a bigger value to $w, \alpha_{n+1}$ is increased as $\alpha_{n}$ is increased. For $\alpha_{n}$ smaller than $0.19 \alpha_{n+1}$ remains 0 , meaning there are no neurons activated at the next time step $n+1$.

\section{Individual Neural Networks with Excitatory Synapses and $r \geq 1$}

Previously, the refractory period of neurons was assumed to be equal to 1 - if of course the neuron receives $P S P \geq \theta$. In general, this restriction does not apply, therefore $r$ should be considered bigger than 1 . In equation (4) the percentage of available neurons for activation is changed, since we have to subtract the percentages of activated neurons not only at the moment $n$ but also at the moments $n-1, n-2, \ldots, n-r$ +1 . So, the equation (4) is transformed to

$$
<a_{n+1}>=\left(1-\sum_{i=n-r+1}^{n} a_{i}\right) * P\left(a_{n}, \theta\right) .
$$


Based on the equation (7) and equation (5), it is possible to calculate the expected value $\alpha_{n+1}$ if the values $\alpha_{n}, \alpha_{n-1}, \alpha_{n-2}, \ldots, \alpha_{n-r+1}$ are known [4]. The network's behavior is investigated while the refractory period $r$ is varied and all the parameters $(\mathrm{N}, \mu, \theta$, w) are maintained constant.

The value of the refractory period does not affect the number of neurons activated at the moment $n+1$ versus the number of neurons activated at the moment $n$ since $r$ now affects more than one time steps. For example, if $r=4,4$ previous time steps of neural activity should be taken into account. To study the affect of $r>1$ on the neuron's network we investigate the evolution of the percentage of active neurons over time. We track the values for which the neural activity is identical, so we calculate the period of the repeated neural activity and the delay that the network needs to reach the periodic activity.

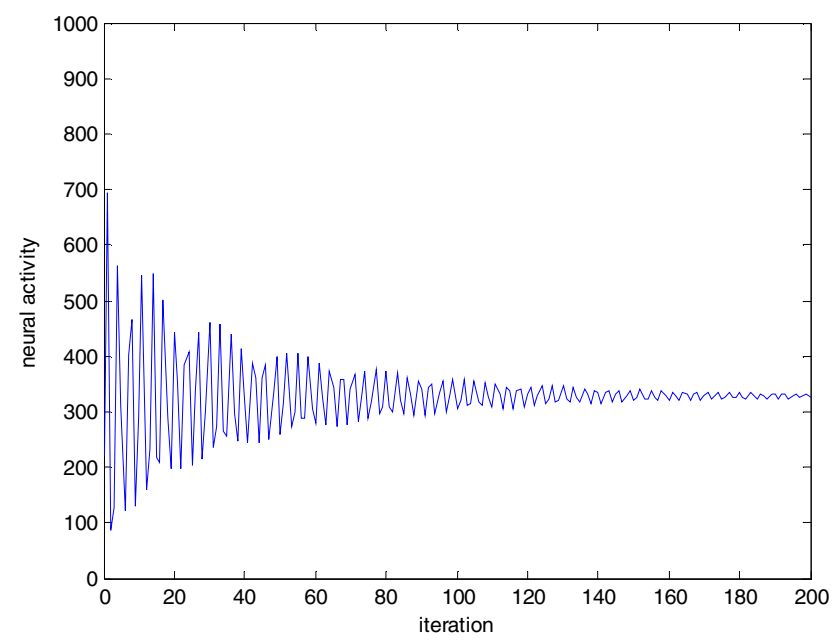

Fig. 5. For $r=2$, the network reaches the periodic activity after 200 iterations and the period is 24 . At the moment where 326 neurons (neural activity) are activated (of the 1000 available) the network reaches a periodic activity.

\section{Asynchronous Individual Neural Networks with Excitatory Synapses and $r \geq 1, \tau \geq 1$}

In this case we split the total number of synapses of the network, which is equal to $\mathrm{N}$ $\times \mu$ into sub-groups of synapses [4]. The synapses that belong to the same sub-group have the same delay $\tau$, which means that $\tau$ time is necessary for PSP to appear in this group of synapses. Suppose that the synapses of the network are split to c sub-groups. Let $f_{j}$ be the percentage of the sub-group of the synapses with synaptic delay $j$. Then

$$
\sum_{j=1}^{c} f_{j}=1
$$


Due to the assumption that the synapses are asynchronous, at the moment $n+1$ the total PSP that is produced is a result of all the neurons that were activated at the moment $n-c+1$ and have synapses with synaptic delay c. If $\mathrm{L}_{n}, \mathrm{~L}_{\mathrm{n}-1}, \mathrm{~L}_{\mathrm{n}-2}, \ldots, \mathrm{L}_{\mathrm{n}-\mathrm{c}+1}$ are the PSPs produced for $\tau=1, \tau=2$, etc. then:

$\mathrm{L}_{\mathrm{n}}=\mathrm{a}_{\mathrm{n}} \times \mathrm{N} \times \mu \times \mathrm{f}_{1}$

$\mathrm{L}_{\mathrm{n}-1}=\mathrm{a}_{\mathrm{n}-1} \times \mathrm{N} \times \mu \times \mathrm{f}_{2}$

$\mathrm{L}_{\mathrm{n}-2}=\mathrm{a}_{\mathrm{n}-2} \times \mathrm{N} \times \mu \times \mathrm{f}_{3}$

and in general :

$$
\mathrm{L}_{\mathrm{j}}=\mathrm{a}_{\mathrm{n}-\mathrm{j}+1} \times \mathrm{N} \times \mu \times \mathrm{f}_{\mathrm{j}} \quad \mathrm{j}=1, \ldots, \mathrm{c} .
$$

If $\mathrm{L}$ is the total PSP produced at the time $\mathrm{n}+1$ then :

$$
L=\sum_{j=n-c+1}^{n} L_{j}=\sum_{j=1}^{c} a_{n-j+1} * N * \mu * f_{j}=N * \mu * \sum_{j=1}^{c} a_{n-j+1} * f_{j}
$$

Based on the L value derived of the equation (10) we may use the equations (1), (2) and (5) to calculate the probability of neurons to be activated. Using the equation (7) the $\alpha_{n+1}$ is calculated for any of the $\mathrm{r}$ and $\tau$ values, given the networks' parameters $\mathrm{N}$, $\mu, \theta, w, r, c$, and $f_{j},(j=1, \ldots, c)$. Below we present the graphs concerning two cases of the network's activity.

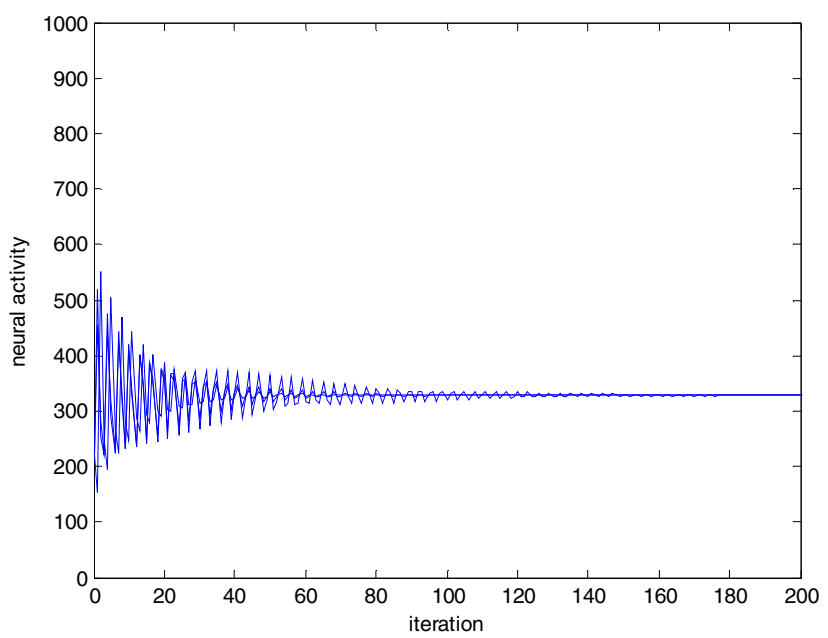

Fig. 6. For 2 sub-groups of synapses and percentage of 0.1 and 0.9 each one. The network reaches a periodic activity after 89 iterations while the period is 1 . When 329 neurons (neural activity) of the 1000 available are activated, the network reaches a periodic activity. 


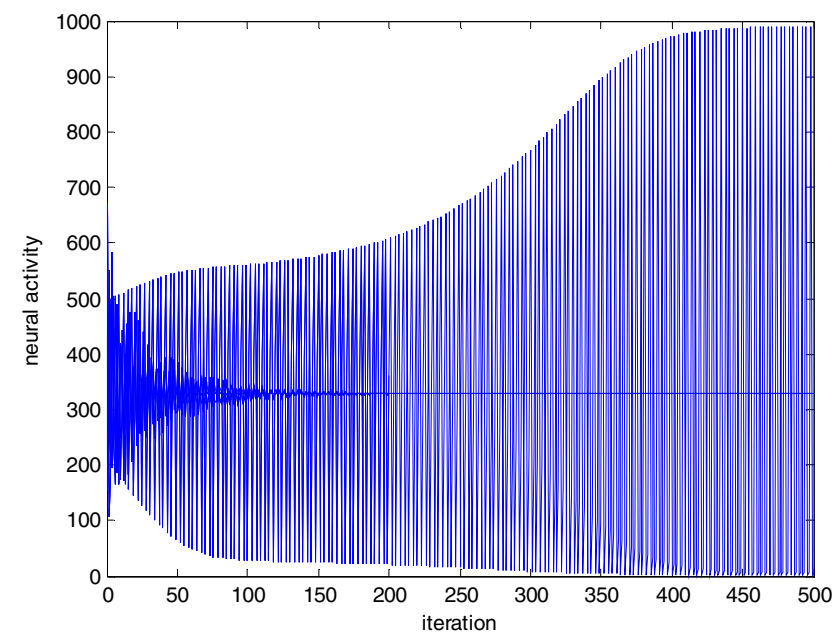

Fig. 7. For 3 subgroups of synapses. The percentage of synapses for each subgroup is $0.1,0.2$ and 0.7 .

We tested the network for more sub-groups with fewer synapses per group and the result was that the bigger the number of the sub-groups, the fewer the number of the neurons are activated initially and fewer are the iterations needed for the network to reach a periodic activity. As it is shown in fig. 7, we tested the network for 500 iterations. The network reaches periodic activity after 480 iterations while the period is 3 . Contrary to the case shown in fig. 6 , where we had 2 sub-groups, in this case more neurons, 990, are activated (neural activity) when the network reaches a periodic activity.

\section{Conclusions}

By studying the aforementioned mathematical models, a few conclusions can be made: The size of the network does not affect the network's behavior, since no matter how many neurons the network is consisted of, its course remains the same. That could mean that we obtain a rather good statistical picture of the network. The number of synapses per neuron affects the network's behavior since when this number is increased, the neurons that are able to spike at the next time step are increased rapidly initially, but eventually this value decreases until it is set to zero. This phenomenon is explained by the network's training when new synapses are created. As far as the triggering threshold is concerned, when it is increased the neurons that are able to spike at the next time step are decreased to zero. For especially big triggering threshold, the neurons are never activated since the treaty $k \geq \theta / w$ (with synaptic weight stable) does not apply. By increasing the synaptic weight, the percentage of neurons that is activated at the next time step is increased rapidly while the percentage of neurons that is activated at the time $\mathrm{n}$ is also increased, remaining zero for a rather small $\alpha_{n}$. For neural networks with refractory period bigger than 1 , we investigate the evolu- 
tion of neurons over time. As the refractory period is increased, many neurons are activated initially, but after a few iterations, they are no longer active. That is reasonable since the probability for the neurons to be active after e.g. 15 time steps is decreased over time and no neuron is activated eventually. Furthermore, the case where both the refractory period and the synaptic delay are bigger than 1 was studied. For this purpose, we split the population of neurons into sub-groups. Each sub-group has various percentages of synapses. Each sub-group had its own synaptic delay (it's the same for the members of the same sub-group). Interestingly, these percentages per subgroup affect the network's activity. If the network is split into a rather big number of subgroups each of which has a small number of synapses, the number of neurons that are activated initially is small. Also, in this case, less iterations are necessary for the system to reach periodic activity.

\section{References}

1. Amit, D.J.: Modelling Brain Function. Cambridge University Press, Cambridge (1989)

2. Trappenberg: Fundamentals of Computational Neuroscience. Oxford University Press, Oxford (2002)

3. Bear, M.F., Connors, B.W., Paradiso, M.A.: Neuroscience: Exploring the Brain, 3rd edn. Lippincott Williams \& Wilkins, Baltimore (2007)

4. Adamopoulos, A.: Introduction to biological neural networks (2007)

5. Giuliodori, M.J., Zuccolilli, G.: Postsynaptic Potential Summation and Action Potential Initiation: Function Following Form. Advan. Physiol. Edu. 28, 79-80 (2004)

6. Eeckman, F.H., Bower, J.M. (eds.): Computation and Neural Systems. Kluwer Academic Publishers, Boston (1993)

7. Bower, J.M.: Modeling the nervous system. Trends Neuroscience 15, 411-412 (1992) 